\title{
Contrast-enhanced Ultrasound Improves Technical Sufficiency of Fine-needle Aspiration in Suspicious Thyroid Nodules
}

\author{
Ying Fu, MD ${ }^{a}$, Shi Tan, MD ${ }^{a}$, LiGang Cui, MD ${ }^{a, *}$, Fang Mei, MD ${ }^{b}$ \\ ${ }^{a}$ Department of Ultrasound, Peking University Third Hospital, Beijing, China; ${ }^{b}$ Department of Pathology, Peking University Third \\ Hospital, School of Basic Medical Sciences, Peking University Health Science Center, Beijing, China \\ Received October 26, 2020; revision received December 08, 2020; accepted December 10, 2020.
}

Objectives: To evaluate contrast-enhanced ultrasound (CEUS) for guiding fine-needle aspiration (FNA) of suspicious thyroid nodules to obtain sufficient biopsy specimens.

Methods: A total of 236 uncertain thyroid nodules detected in 228 patients from October 2016 to March 2017 were retrospectively reviewed and analyzed in this study. Overall, 117 patients underwent CEUS-guided FNA, and 111 patients underwent ultrasound (US)-guided FNA. The target for aspiration was only at the enhanced area in the CEUS group. In the USguided group, aspiration was conducted within the nodule at various angles and areas. The final cytopathologic findings were reported using the Bethesda criteria. The inadequacy, indeterminacy, malignancy, and benignity rates of FNA specimens were compared between two groups.

Results: There were no significant differences in age, sex, or nodule size between the two groups. The inadequacy rate in the CEUS group was significantly lower than that in the US group $(P=0.008)$. Twenty-two benign nodules were diagnosed using CEUS-guided FNA, whereas seven were diagnosed using US-guided FNA $(P=0.006)$. The indeterminacy and malignancy rates were similar for both groups.

Conclusions: CEUS-guided FNA improves the diagnostic success rate and reduces uncertainty by facilitating accurate biopsy of suspected thyroid nodules with microcirculation perfusion imaging.

Key words: Thyroid; Neoplasm; Ultrasound; Contrast agent; Fine-needle aspiration

Advanced Ultrasound in Diagnosis and Therapy 2021; 03: 219-225

DOI: 10.37015/AUDT.2021.200063

$\mathrm{C}$ linicians are routinely expected to manage patients with thyroid nodules. For primary care, thyroid nodules are detected in up to $50-60 \%$ of healthy individuals [1]. However, most nodules are clinically asymptomatic, and only $15 \%$ of patients with thyroid nodules are malignancy. Thyroid fine-needle aspiration (FNA) is the first-line diagnostic modality for the evaluation of thyroid nodules. It is recommended as the most essential and primary diagnostic tool to distinguish malignant from benign thyroid nodules in almost all practice guidelines [2].

Even with an experienced operator under ultrasound (US) guidance, approximately $10-40.7 \%$ of FNA procedures produce a non-diagnostic result, with a $1-4 \%$ estimated risk of malignancy [2-4]. However, surgical resection is performed in $16.2 \%$ of cases with a nondiagnostic result [4].

Several studies have evaluated the factors affecting the diagnostic outcomes of US-guided FNA of thyroid nodules $[5,6]$. US-FNA strongly depends on the operator,

\footnotetext{
* Corresponding author: Department of Ultrasound, Peking University Third Hospital, No. 49 North Garden Road, Haidian District, Beijing 100191, China

e-mail: cuijuegang@126.com unrestricted use, distribution and reproduction in any medium provided that the original work is properly attributed.
} 
cytopathologist, and the intrinsic nodule characteristics [3]. Cystic lesions are most commonly responsible for nondiagnostic results [7]. Various studies have evaluated the cystic content of each nodule [5]. Cystic content is usually attributable to recent and old hemorrhage, cystic degeneration, or necrosis. It is not easy for the conventional US (even with high-frequency probes) to identify the presence of necrotic tissue, particularly if liquefactive necrosis does not occur. Therefore, inappropriate localization of the site for FNA may be the reason for non-diagnostic sampling.

Color Doppler flow imaging (CDFI) is a safe and inexpensive examination to detect the vascularity of the nodule. However, in our experience and literature, it cannot detect necrosis as $33 \%$ of malignant thyroid nodules have no vascular flow [8].

Currently, few studies consider US guidance for FNA to be superior to the free-hand technique [1-3]. Recently, contrast-enhanced ultrasound (CEUS) has increased clinical use in the liver and small organs [9-11]. It has been widely used for tumor diagnosis and is a valuable technique for visualizing macro-and microcirculation with the depiction of tumor vascularization. In addition, ultrasound contrast media does not show renal, hepatic, or cardiac toxicity and does not influence the thyroid gland [10].

We hypothesize that CEUS can increase the diagnostic effectiveness of FNA for suspected thyroid nodules with a decreased inadequacy rate and more abundant cell count. Thus, the aim of this study was to evaluate CEUS-guided FNA of suspicious thyroid nodules to obtain sufficient biopsy specimens and compare to only US-guided FNA.

\section{Materials and Methods}

We retrospectively analyzed patients who underwent FNA at our institution between October 2016 and March 2017. The study protocol was approved by our institutional review board. Written informed consent was obtained from all enrolled patients.

\section{Patients}

Study subjects included 228 consecutive patients (50 males and 178 females; age: $23-81$ years; mean age: 45 years) with 236 uncertain thyroid nodules detected either on conventional US or computed tomography (CT) who underwent FNA in our center. In our center, FNA was performed with CEUS from January 2017, so patients were divided into two groups based on time point: US group (from October 2016 to December 2016, the patients underwent unenhanced US before FNA) and CEUS group (January 2017 to March 2017, the patients underwent CEUS before FNA).

Patients in the US group did not undergo CEUS before conventional US-guided biopsy. We performed FNA on thyroid nodules $\geqslant 5 \mathrm{~mm}$, with intermediate suspicion or high suspicion. Patients with thyroid nodules who underwent ablation before FNA were excluded. The largest two nodules suspected of malignancy were selected as the target lesions when multiple nodules were detected. We did not biopsy more than two nodules from the same patient at the same time[1].

The inclusion criteria for FNA were as follows: (a) age $\geqslant 18$ years; (b) thyroid nodules visible on ultrasound; and (c) nodule diameter $\geqslant 5 \mathrm{~mm}$, with intermediate suspicion or high suspicion based on TIRADS classification[12].

The exclusion criteria were (a) patients with serious bleeding tendency and (b) patients with severe cough or heart failure who could not tolerate supine during the puncture process.

\section{CEUS examination}

Patients in the CEUS group underwent CEUS before conventional US-guided FNA. The sonographic examinations were performed using two commercially available US scanners (Logic E9, GE Healthcare, Chicago, IL; Siemens ACUSON S3000, Siemens Healthineers, Erlangen, Germany) for conventional US, and an L9 transducer (Siemens Healthineers and GE Healthcare) for CEUS. The location, number, size, and ultrasonographic features of suspected nodules were evaluated and recorded. Subsequently, a contrast-specific imaging mode was used. The 2.4-3.0 mL SonoVue (Bracco, Milan, Italy) was administered intravenously through the antecubital vein within 2-3 s. The perfusion and enhancement patterns of the target lesions were continuously observed and recorded throughout all dynamic phases of CEUS. If there was no enhancement area in the nodule, the target for aspiration was aimed at the enhanced area only (Fig. 1, Fig. 2).

\section{Comparison of US-guided and CEUS-guided FNAs}

The FNAs were performed by the experienced radiologists, who performed $>100$ FNAs/year (one engaged in biopsy for 10 years, and the other for 11 years). A $25-G$ needle (Hakko, Chikuma, Japan) was used under US guidance, and 4 passes were made into each nodule. Each pass included 6-7 "to-and-fro" needle movements over 5-10 s for the nodule. We performed CDFI on the target nodule and peripheral parenchyma to avoid bold arteries. In the US group, aspiration was conducted at different angles and regions within the nodule to ensure a representative sample obtained. In the CEUS group, the target of aspiration was the enhanced area only. The sample was obtained mainly via the "capillary method" (without suction). The aspirated 
Fu et al. CEUS improves sufficiency of thyroid nodule FNA

material was submerged in a single vial of methanolbased preservative solution (Cytoprep preservative solution; Cheng Zhi Guang Hui, Beijing, China), and sent to the cytopathology laboratory for centrifugation and staining (SurePath; TriPath Imaging, Burlington, NC). There was one slide for each lesion; each slide had a thin layer of cells concentrated on the central area of the slide. The specimens were not reviewed on-site for adequacy.

\section{Cytopathology}

The experienced pathologist blinded to the guiding method and reported the cytopathology findings according to the Bethesda criteria [13]: non-diagnostic (less than 6 groups of well-visualized follicular cells, each group containing less than 10 well-preserved epithelial cells), benign, atypia of undetermined significance/follicular lesion of undetermined significance (AUS/FLUS), follicular neoplasm/suspicious for follicular neoplasm (FN/SFN), suspicious for malignancy, and malignancy. The number of cells in the specimens was classified into two classes according to the cytopathologist's experience: intermediate (possible diagnosis) and abundant (probable diagnosis).

\section{Statistical analysis}

The data were expressed as mean \pm standard deviation. Continuous variables were tested by analysis of variance (ANOVA). Categorical variables were analyzed by the chi-squared test. The diagnosis of thyroid nodules was as follows: (1) surgery, (2) histopathologic and corresponding follow-up results (at least 1 year), (3) evidence of either benign or malignant cytology on FNA and on the corresponding follow-up result. The thyroid FNA biopsies were evaluated by inadequacy, indeterminacy, malignancy, and benignity rates, respectively. The inadequacy rate refers to the proportion of cases with non-diagnostic results. The indeterminate rate refers to the proportion of cases with an AUS or FLUS category. The malignancy rate refers to the proportion of malignant cases. The benignity rate refers to the proportion of benign cases. All tests were performed SPSS version 22.0 (IBM, Armonk, NY), with $P<0.05$ indicating statistical significance.

\section{Results}

\section{Participant characteristics}

In the US group, 113 thyroid nodules were biopsied. In the CEUS group, 123 nodules were biopsied. There were no significant differences in age, sex, or nodule size between the two groups $(P>0.05)$. Table 1 showed the clinical characteristics of the two groups.
Table 1 The clinical characteristics of 228 patients with suspicious malignant thyroid nodules

\begin{tabular}{lccc}
\hline Characteristic & US group & CEUS group & $P$ value \\
\hline Age (years) & $45.54 \pm 13.8$ & $43.72 \pm 12.93$ & 0.305 \\
Sex (female/male) & $88 / 23$ & $90 / 27$ & 0.667 \\
\hline Nodule size (cm) & $1.08 \pm 0.53$ & $1.18 \pm 0.56$ & 0.170 \\
\hline
\end{tabular}

US, ultrasound; CEUS, contrast-enhanced ultrasound.

\section{Bethesda categories for each group}

Table 2 showed the results of 2 groups based on Bethesda categories. Of the 236 thyroid nodules with FNA, the inadequacy rate in the CEUS group was significantly lower than that in the US group (4/123 vs. $14 / 113, P<0.01)$. The proportions of diagnosed malignant nodules in the CEUS group (75/123) and the US group (73/113) were similar. Twenty-two benign nodules $(14.6 \%)$ were diagnosed with CEUS-guided FNA, while 7 (6.2\%) were diagnosed with US-guided FNA. The benignity rate in the CEUS group was significantly higher than that in the US group $(P=0.006)$ (Fig. 2). There was no significant difference in the indeterminate rate between the two groups (20/113 vs. $22 / 123, P=0.970)$. The cell count in the diagnosable specimens, classified as intermediate (possible diagnosis) and abundant (probable diagnosis), did not show a significant difference between the two groups $(P=0.096)$ (Table 3).

Table 2 Distributions of Bethesda categories for in each group

\begin{tabular}{lccc}
\hline Bethesda category & $\begin{array}{c}\text { US group } \\
\text { (nodule) }\end{array}$ & $\begin{array}{c}\text { CEUS group } \\
\text { (nodule) }\end{array}$ & $P$ value \\
\hline Non-diagnostic & $14(12.4 \%)$ & $4(3.3 \%)$ & 0.008 \\
\hline Benign & $7(6.2 \%)$ & $22(17.9 \%)$ & 0.006 \\
\hline AUS/FLUS & $7(6.2 \%)$ & $12(9.8 \%)$ & 0.315 \\
\hline FN/SFN & $13(11.5 \%)$ & $10(8.1 \%)$ & 0.383 \\
\hline Suspicious for malignancy & $13(11.5 \%)$ & $15(12.2 \%)$ & 0.870 \\
\hline Malignancy & $59(52.2 \%)$ & $60(48.8 \%)$ & 0.598 \\
\hline Total & 113 & 123 & \\
\hline
\end{tabular}

US, ultrasound; CEUS, contrast-enhanced ultrasound; AUS/FLUS, atypia of undetermined significance/follicular lesion of undetermined significance; FN/SFN, follicular neoplasm/suspicious for follicular neoplasm.

Table 3 The classification of the cell count for the diagnosable thyroid nodules

\begin{tabular}{lccc}
\hline \multirow{2}{*}{ Group } & \multicolumn{2}{c}{ Cell amount } & \multirow{2}{*}{$P$ value } \\
\cline { 2 - 3 } & Intermediate & Abundant & \\
\hline US group & 69 & 30 & 0.096 \\
CEUS group & 70 & 49 & \\
\hline
\end{tabular}

US, ultrasound; CEUS, contrast-enhanced ultrasound. 



Figure 1 A 29-year-old woman with a non-palpable mass in the right lobe of the thyroid. Surgical pathology confirmed the presence of a papillary thyroid carcinoma with necrosis. (A) Ultrasound showed a hypoechoic mass measuring $14 \mathrm{~mm} \times 8 \mathrm{~mm}$ with an ill-defined margin in the longitudinal section; (B) The nodule showed a few amounts of blood flow signal on color Doppler. (C) Contrast-enhanced ultrasound showed heterogeneous enhancement; (D) After fine needle aspiration at the area of enhancement area, the nodule was diagnosed as "malignant" with abundant cell amount(magnification $\times 20$ ).

Table 4 Characteristics of largest nodule diameters between groups

\begin{tabular}{|c|c|c|c|c|}
\hline \multirow{2}{*}{$\begin{array}{l}\text { Largest nodule } \\
\text { diameter in different } \\
\text { groups }\end{array}$} & \multirow{2}{*}{$\begin{array}{l}\text { Number of } \\
\text { nodules }\end{array}$} & \multicolumn{2}{|c|}{$\begin{array}{c}\text { Cytopathology result/ } \\
\text { number }\end{array}$} & \multirow{2}{*}{$P$ value } \\
\hline & & $\begin{array}{c}\text { Non- } \\
\text { diagnostic }\end{array}$ & Diagnostic & \\
\hline$<1 \mathrm{~cm}$ in US group & 57 & 8 & 49 & 0.139 \\
\hline$<1 \mathrm{~cm}$ in CEUS group & 51 & 2 & 49 & \\
\hline$\geqslant 1 \mathrm{~cm}$ in US group & 56 & 6 & 50 & 0.141 \\
\hline $\begin{array}{l}\geqslant 1 \mathrm{~cm} \text { in CEUS } \\
\text { group }\end{array}$ & 72 & 2 & 70 & \\
\hline
\end{tabular}

US, ultrasound; CEUS, contrast-enhanced ultrasound.
The mean diameter in the US group for the nondiagnostic result was $1.19 \pm 0.6 \mathrm{~cm}$, while that in the CEUS group was $1.08 \pm 0.48 \mathrm{~cm}(P=0.739)$. The distributions of the largest nodule diameters in each group were shown in Table 4.

Moreover, CEUS-guided FNA detected five new nodules that were missed by US. These missed nodules were confirmed to be malignant, according to FNA and surgical results. Five patients who received alterations to their surgery plan underwent total thyroidectomy for complete resection of the multi-centric disease and compartmental node dissection, as opposed to lobectomy only.


Figure 2 A 53-year-old woman with a non-palpable nodule in the left lobe of the thyroid. Cytopathologic findings confirmed the presence of a benign nodule. (A) Gray-scale ultrasound showed an isoechoic mass measuring $12 \mathrm{~mm} \times 7 \mathrm{~mm}$ with an obscure border; a thick, incomplete, non-uniform halo was evident in the transverse section. Color Doppler ultrasound showed no blood signals in the same section; (B) Contrast-enhanced ultrasound showed homogeneous enhancement at the center of the nodule. There was no enhancement at the peripheral area of the nodule; (C) The area of enhancement was aspirated, and it was diagnosed as a benign lesion (magnification $\times 20$ ). As of writing, this nodule did not show any sign of enlargement. 

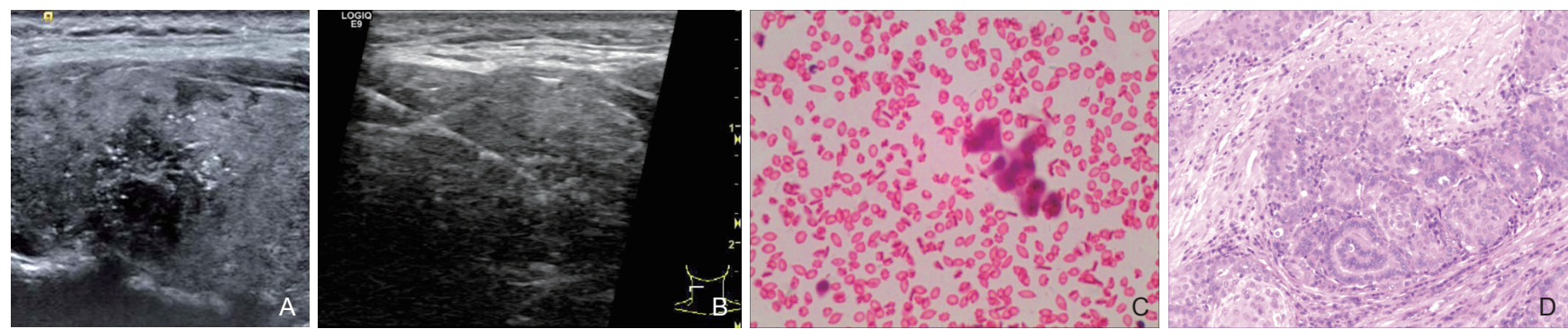

Figure 3 A 29-year-old woman with a non-palpable mass in the right lobe of the thyroid; results indicated non-diagnostic cytological findings on ultrasound-guided fine-needle aspiration (FNA). (A) Gray-scale ultrasound showed a hypoechoic mass measuring $18 \mathrm{~mm} \times 14 \mathrm{~mm}$ indicating the presence of multiple microcalcifications. On color Doppler flow imaging, the nodule showed sparse vascularity; (B) FNA was performed on the nodule; (C) There was no diagnostic result following cytology; (D) However, the patient received surgery because the nodule showed high suspicion of malignancy on ultrasound. Eventually, the patient was diagnosed as having a diffuse sclerosing variant of papillary thyroid carcinoma.

Overall, 175 patients underwent surgery, and the remaining 53 patients were still awaiting follow-up at the time of writing. Nodules have shown no tumor enlargement to date. 114 patients were diagnosed as malignancy via surgery. Only one was misdiagnosed; The patient, who had been previously diagnosed with FLUS by FNA, was re-diagnosed with a diffuse sclerosing variant of papillary thyroid carcinoma by surgery (Fig. 3).

\section{Discussion}

FNA of thyroid nodules has reduced the need for unnecessary surgical procedures by $25 \%$ over the past several decades [14,15]. However, as per a previous study, $2-20 \%$ of FNAs were qualitatively or quantitatively insufficient to establish a definite diagnosis [4]. Owing to the underlying risk of malignancy, most guidelines recommend performing a repeat FNA under US guidance for non-diagnostic FNAs [1-4]. However, approximately one-third of the patients who undergo FNA with non-diagnostic results will be lost to followup [16]. Furthermore, repeat aspiration may increase patients' discomfort and anxiety and may incur higher medical expenses. The categories of AUS/FLUS and FN/SFN, referred to as the "gray zone," include both benign and malignant outcomes, which pose a difficult challenge for clinical management. Understanding the nature of "gray zone" nodules will help refine diagnostic and therapeutic strategies. Therefore, the first cytologic specimen from FNA must have adequate cellularity and a satisfactory count of representative follicular epithelial cells.

Guidelines for liver CEUS indicate that the performance of this procedure before a biopsy can increase the diagnostic yield by $10 \%$ as well as decrease the false-negative rate, particularly in large tumors with areas of necrosis, in both cirrhotic and normal livers. CEUS can identify the most appropriate site for a biopsy more accurately than US; it does this by visualizing the regions of the vascularized, viable tumor that should be targeted, while also identifying the necrotic regions that should be avoided [17]. Wu et al. reported the utility of CEUS for percutaneous biopsies of focal hepatic lesions; a single puncture attempt was successful in $14.0 \%$ of the patients in the CEUS group as compared to $4.9 \%$ of the patients who underwent B-mode US guidance [18]. Eso et al. reported that sonazoid-enhanced US guidance improved the accuracy of pathological diagnoses based on biopsies of focal hepatic lesions by providing location information of the enhanced viable area in the vascular phase; this permitted the avoidance of unenhanced avascular fibrotic and necrotic areas [19].

Inspired by the successful application of CEUS for guided biopsies of liver nodules, we investigated whether CEUS could be used to guide FNAs for thyroid nodules. In our current study, the diagnostic success rate (adequacy) of FNAs in the CEUS group was significantly higher than that in the US group (95.3\% vs. $87.4 \%)$. This finding was consistent with the results obtained with the use of CEUS to the liver $[18,19]$. For an indepth analysis, we classified the number of cells in the diagnosable specimens as either intermediate (possible diagnosis) or abundant (probable diagnosis). Although there were no significant differences between the two groups in terms of indeterminacy rate or cell number, the CEUS group had a significantly higher number of benign nodules ( 22 vs. 7 ). This result allowed us to identify patients who would only require regular follow-up due to the benign diagnosis.

In addition, CEUS could help locate the lesions invisible on non-enhanced US. Five nodules that had been missed on US were revealed by CEUS, and the lesions were confirmed to be malignant. In our hospital, total thyroidectomy was performed for patients with two or more malignant nodules, while lateral lobectomy was performed on patients with a single malignant thyroid nodule. Consequently, these five patients underwent total thyroidectomy and compartmental node dissection, in which CEUS provided useful information for clinical 
management and surgical decision making process.

Previous studies have reported the utility of CEUS for percutaneous core biopsies of focal thyroid lesions. $\mathrm{Li}$ et al. evaluated the diagnostic value of CEUS-guided and US-guided thyroid puncture biopsy in 48 patients with 51 solid thyroid nodules (suspected papillary thyroid carcinoma) [20]. Compared with conventional US, a greater number of papillary thyroid carcinomapositive nodules were detected by CEUS, with increased sensitivity and accuracy of the puncture points $(82.9 \%$ and $82.6 \%$, respectively). By puncturing the highenhancement areas and peripheral areas with rich blood supply, Li et al. could achieve 100\% satisfaction rate for tissue drawing. However, there have limited reports on the use of CEUS guidance for FNAs. Our study evaluated and validated the application of CEUS guidance for the biopsies of thyroid nodules.

The increased diagnostic rate of FNAs in the CEUS group may be attributed to the accurate identification of cystic or hemorrhagic regression areas within the nodule. Several factors may be related to the inadequate sample rate and the US appearance of the thyroid nodule, including the operator's experience, use of palpation guidance techniques as opposed to US guidance, techniques used for cytologic preparation, and interpretation of the FNA specimens [3,21]. In our study, most of these factors were comparable between the two groups, such as the nodule size, ratio of benign and malignant nodules, operation method, and operator experience. However, this was a retrospective study.

These findings show that CEUS is a key factor in acquiring additional information about the nodule. Conventional US alone cannot identify small areas of necrotic tissue, particularly if areas of liquefactive and necrotic tissue are absent upon sequencing. In contrast, a necrotic region usually showed no enhancement in the vascular phases after SonoVue injection, but was easily visualized as an echo-free or slightly hypoechoic signal area when compared to the highly enhanced thyroid parenchyma.

Moreover, the appearance of cystic changes in benign thyroid nodules is referred to as a process of mummification, resulting in a misleading diagnosis of malignancy on US [22]. The FNA results of mummified nodules are usually non-diagnostic or present atypia of unknown significance, which would lead to an unnecessary thyroid surgery or unwarranted anxiety. In such cases, CEUS plays a key role in differentiating viable tissue from necrotic tumor regions and in targeting the puncture point within the nodule.

This study has several limitations. First, the retrospective study design may be considered an important limitation. Second, because of the small sample size used in this study, the results may not be generalizable to a relatively large patient cohort. A wellcontrolled, generalizable, randomized trial is therefore warranted to provide more definitive evidence for the role of CEUS before FNAs. Third, the non-diagnostic specimens, which included abundant colloid and few epithelial cells, most likely would result in a benign diagnosis, consistent with the CEUS result. In the new Bethesda classification, these findings could be reclassified differently into a benign lesion [2,23].

\section{Conclusion}

In conclusion, this study highlights that the performance of CEUS prior to FNA can help to reduce the non-diagnostic rate and improve the benignity rate by allowing for the accurate aspiration of suspected thyroid nodules with microcirculation perfusion imaging. Moreover, CEUS may also help to locate occult lesions on non-enhanced US. These results suggest that the clinical value of CEUS before FNA should be investigated further in future large, randomized, prospective studies.

\section{Conflicts of Interest}

All authors certify that there are no actual or potential conflicts of interest in this article.

\section{References}

[1] Gharib H, Papini E, Garber JR, Duick DS, Harrell RM, Hegedüs L, et al. AACE/ACE/AME Task force on thyroid nodules. American Association of Clinical Endocrinologists, American College of Endocrinology, and Associazione Medici Endocrinologi Medical Guidelines for Clinical Practice for the Diagnosis and Management of Thyroid Nodules--2016 Update. Endocr Pract 2016; 22: 622-639.

[2] Haugen BR, Alexander EK, Bible KC, Doherty GM, Mandel SJ, Nikiforov YE, et al. 2015 American Thyroid Association management guidelines for adult patients with thyroid nodules and differentiated thyroid cancer: the American Thyroid Association guidelines task force on thyroid nodules and differentiated thyroid cancer. Thyroid 2016; 26: 1-133.

[3] Lee YH, Baek JH, Jung SL, Kwak JY, Kim JH, Shin JH et al. Ultrasound-guided fine needle aspiration of thyroid nodules: a consensus statement by the Korean society of thyroid radiology. Korean J Radiol 2015; 16: 391-401.

[4] Bongiovanni M, Spitale A, Faquin WC, Mazzucchelli L, Baloch ZW The bethesda system for reporting thyroid cytopathology: a metaanalysis. Acta Cytol 2012; 56: 333-339.

[5] Alexander EK, Heering JP, Benson CB, Frates MC, Doubilet PM, Cibas ES, et al. Assessment of non-diagnostic ultrasound-guided fine needle aspirations of thyroid nodules. J Clin Endocrinol Metab 2002; 87: 4924-4927.

[6] Choi SH, Han KH, Yoon JH, Moon HJ, Son EJ, Youk JH, et al Factors affecting inadequate sampling of ultrasound guided fineneedle aspiration biopsy of thyroid nodules. Clin Endocrinol (Oxf) 2011; 74: 776-782

[7] Fadda G, Rossi ED. Liquid-based cytology in fine-needle aspiration 
biopsies of the thyroid gland. Acta Cytol 2011; 55: 389-400.

[8] Khadra H, Bakeer M, Hauch A, Hu T, Kandil E. Is vascular flow a predictor of malignant thyroid nodules? a meta-analysis. Gland Surg 2016; 5: 576-582.

[9] Xia HS, Wang X, Ding H, Wen JX, Fan PL, Wang WP. Papillary breast lesions on contrast-enhanced ultrasound: morphological enhancement patterns and diagnostic strategy. Eur Radiol 2014; 24 : 3178-3190.

[10] Müller-Peltzer K, Rübenthaler J, Reiser M, Clevert DA. Contrastenhanced ultrasound (CEUS) of the liver: Critical evaluation of use in clinical routine diagnostics. Radiologe 2017; 57: 348-355.

[11] Solbiati L, Ierace T, Tonolini M, Cova L. Guidance and monitoring of radiofrequency liver tumor ablation with contrast-enhanced ultrasound. Eur J Radiol 2004; 51 Suppl: S19-23.

[12] Tessler FN, Middleton WD, Grant EG, Hoang JK, Berland LL, Teefey SA, et al. ACR Thyroid Imaging, Reporting and Data System (TI-RADS): White Paper of the ACR TI-RADS Committee. $J$ Am Coll Radiol 2017; 14: 587-595.

[13] Ali SZ, Cibas ES. The bethesda system for reporting thyroid cytopathology. Am J Clin Pathol 2009; 132: 658-665.

[14] Rossi ED, Bizzarro T, Martini M, Straccia P, Lombardi CP, Pontecorvi A, et al. The role of fine-needle aspiration in the thyroid nodules of elderly patients. Oncotarget 2016; 7: 11850-11859.

[15] Gharib H, Goellner JR. Fine-needle aspiration biopsy of the thyroid: an appraisal. Ann Intern Med 1993; 118: 282-289.

[16] Espinosa De Ycaza AE, Lowe KM, Dean DS, Castro MR, Fatourechi $\mathrm{V}$, Ryder M, et al. Risk of malignancy in thyroid nodules with non- diagnostic fine-needle aspiration: a retrospective cohort study. Thyroid 2016; 26: 1598-1604.

[17] Claudon M, Dietrich CF, Choi BI, Cosgrove DO, Kudo M, Nolsøe $\mathrm{CP}$, et al. Guidelines and good clinical practice recommendations for contrast enhanced ultrasound (CEUS) in the liver - update 2012: A WFUMB-EFSUMB initiative in cooperation with representatives of AFSUMB, AIUM, ASUM, FLAUS and ICUS. Ultrasound Med Biol 2013; 39: 187-210.

[18] Wu W, Chen MH, Yin SS, Yan K, Fan ZH, Yang W, et al. The role of contrast-enhanced sonography of focal liver lesions before percutaneous biopsy. AJR Am J Roentgenol 2006; 187: 752-761.

[19] Eso Y, Takai A, Takeda H, Matsumoto T, Lee M, Inuzuka T, et al. Sonazoid-enhanced ultrasonography guidance improves the quality of pathological diagnosis in the biopsy of focal hepatic lesions. Eur J Gastroenterol Hepatol 2016; 28: 1462-1467.

[20] Li F, Luo H. Comparative study of thyroid puncture biopsy guided by contrast-enhanced ultrasonography and conventional ultrasound. Exp Ther Med 2013; 5: 1381-1384.

[21] Choi SH, Han KH, Yoon JH, Moon HJ, Son EJ, Youk JH, et al Factors affecting inadequate sampling of ultrasound-guided fineneedle aspiration biopsy of thyroid nodules. ClinEndocrinol (Oxf) 2011; 74: 776-782.

[22] Lacout A, Chevenet C, Marcy PY. Mummified thyroid syndrome. AJR Am J Roentgenol 2016; 206: 837-845.

[23] Cibas ES, Ali SZ. The 2017 bethesda system for reporting thyroid cytopathology. Thyroid 2017; 27: 1341-1346. 